\title{
REATIVIDADE LINFONODAL COM METÁSTASE CERVICAL DE TUMOR PRIMÁRIO OCULTO - ESTUDO DE 24 PACIENTES
}

\author{
LYMPHONODE REACTIVITY WITH CERVICAL METASTASIS FROM \\ UNKNOWN PRIMARY TUMOR - STUDY OF 24 PATIENTS.
}

\author{
Ali Amar ${ }^{1}$ \\ Marilene Paladino Rosa ${ }^{2}$ \\ Abrão Rapoport, TCBC-SP 3 \\ Marcos Ribeiro Magalhães ${ }^{1}$ \\ Marcos Brasilino de Carvalho ${ }^{4}$
}

\begin{abstract}
RESUMO: Objetivo: Avaliar a reatividade linfonodal em pacientes com metástase cervical de tumor primário oculto. Método: Foram avaliados retrospectivamente 24 pacientes submetidos a esvaziamento cervical entre 1983 e 1995 devido à metástase de tumor primário oculto. Os cortes histológicos dos 601 linfonodos resultantes foram corados pela hematoxilina-eosina e avaliados à microscopia óptica. A reatividade linfonodal considerou a presença de hiperplasia paracortical, hiperplasia de centro germinativo e hiperplasia sinusal. Foram avaliadas a relação da reatividade linfonodal, ruptura capsular, necrose e desmoplasia com a recidiva da doença. A análise estatística foi feita com Teste de Fisher com erro inferior a 5\%. Resultados: Setenta e sete por cento (77\%) dos linfonodos se mostraram reativos. Cinqüenta e cinco por cento $(55 \%)$ dos pacientes com hiperplasia paracortical ou mista e dezesseis por cento (16\%) dos pacientes com hiperplasia de centro germinativo ou linfonodos não reativos estavam assintomáticos por ocasião do último retorno ambulatorial $(p=0,11)$. A presença de necrose tumoral mostrou associação significativa com a presença de desmoplasia $(\mathrm{p}=0,02)$. Conclusões: A reatividade linfonodal é frequiente na maioria das metástases cervicais em tumor primário oculto e a necrose tumoral está diretamente ligada à presença de desmoplasia.
\end{abstract}

Descritores: Primário oculto; Linfonodo; Metástase; Câncer.

\section{INTRODUÇÃO}

A metastatização é a principal característica clínica das neoplasias malignas, sendo que os linfonodos hiperplásicos são freqüentemente encontrados durante os esvaziamentos cervicais, com significado clínico ainda não elucidado.

Black et al. ${ }^{1}$, após observarem histiocitose no baço de ratos que haviam apresentado regressão tumoral espontânea, encontraram relação entre a histiocitose em lin- fonodos provenientes de esvaziamento axilar e o prognóstico em pacientes com câncer de mama. Apesar das diversas tentativas no sentido de correlacionar as alterações morfológicas nos linfonodos com a metastatização e a evolução clínica de pacientes com câncer, o assunto ainda é controverso ${ }^{1-4}$.

Os tumores primários ocultos correspondem a aproximadamente $3,8 \%$ dos tumores das vias aerodigestivas superiores ${ }^{5}$. Em poucos pacientes se identifica posteriormente uma lesão primária, mesmo naqueles tratados uni-

1. Médico Assistente do Serviço de Cirurgia de Cabeça e Pescoço e Mestre pelo Curso de Pós-Graduação em Cirurgia de Cabeça e Pescoço do Complexo Hospitalar Heliópolis-SP.

2. Médica Patologista do Serviço de Cirurgia de Cabeça e Pescoço do Complexo Hospitalar Heliópolis - SP.

3. Coordenador do Curso de Pós-Graduação em Cirurgia de Cabeça e Pescoço do Complexo Hospitalar Heliópolis-SP.

4. Chefe do Serviço de Cirurgia de Cabeça e Pescoço do Complexo Hospitalar Heliópolis-SP.

Recebido em 16/8/2000

Aceito para publicação em 19/12/2000

Trabalho realizado no Serviço de Cirurgia de Cabeça e Pescoço do Complexo Hospitalar Heliópolis, Hosphel, São Paulo - SP. 
camente com esvaziamento cervical, com uma incidência similar à observada no aparecimento de segundo tumor ${ }^{6}$.

Este estudo tem por objetivo avaliar a presença e o significado clínico da reatividade dos linfonodos em pacientes com metástase cervical de tumor primário oculto.

\section{MÉTODO}

Foram avaliados retrospectivamente 24 pacientes portadores de metástase cervical de tumor primário oculto atendidos no Serviço de Cirurgia de Cabeça e Pescoço do Hospital Heliópolis de 1983 a 1995. Foram incluídos os pacientes que se submeteram a esvaziamento cervical, com seguimento mínimo de 12 meses para os pacientes assintomáticos, cujos cortes histológicos estivessem disponíveis para revisão. O estadiamento empregou os critérios definidos pela classificação TNM da UICC/AJC-1997.

Os cortes histológicos dos linfonodos metastáticos e não metastáticos, corados pela hematoxilina-eosina, foram avaliados por microscopia óptica. A definição do padrão de reatividade se baseou nos critérios definidos por Tsakraklides et $\mathrm{al}^{3}$, com algumas modificações discriminadas a seguir:

1. Linfonodo com hiperplasia folicular: hiperplasia de centros germinativos na cortical do linfonodo, com a formação de folículos secundários (Figura 1).

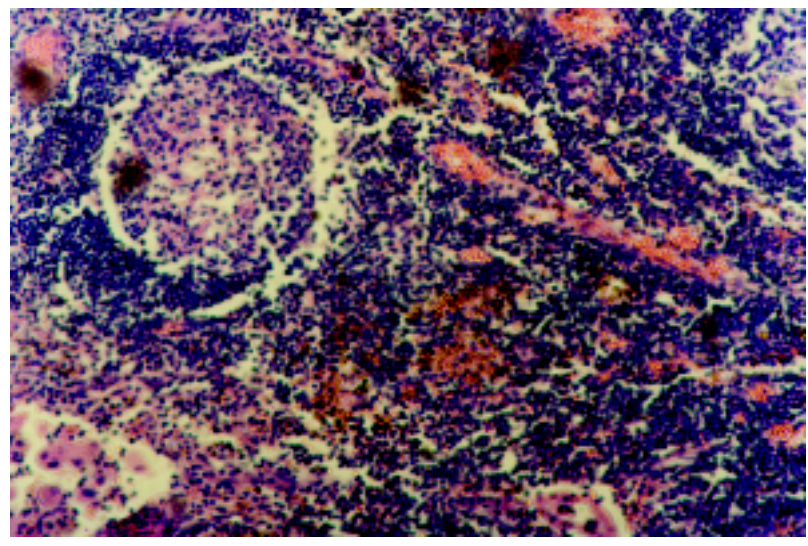

Figura 1 - Hiperplasia folicular (HE, 125x).

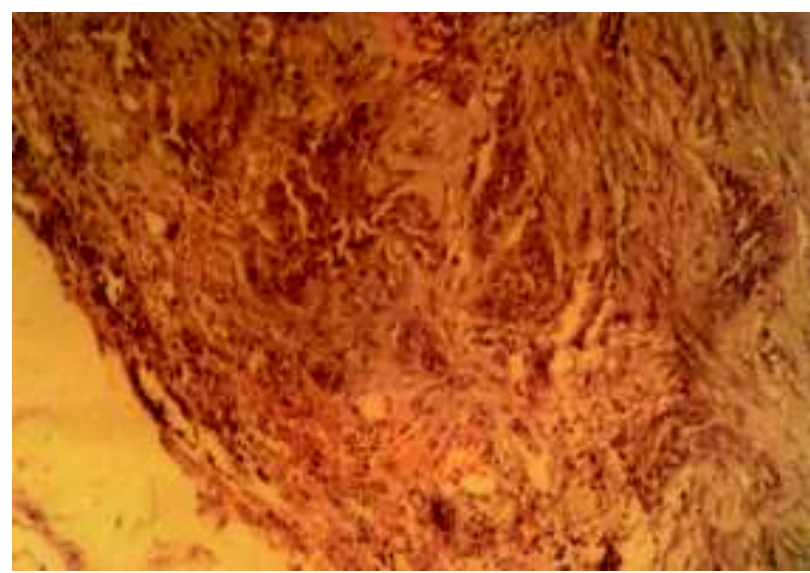

Figura 3 - Linfonodo não estimulado (HE, 125x).
2. Linfonodo com hiperplasia paracortical: aumento de celularidade (linfócitos) na área paracortical (Figura 2).

3. Linfonodo não estimulado: linfonodos sem proliferação linfóide (Figura 3).

4. Linfonodo com a hiperplasia sinusal e histiocitose. A hiperplasia sinusal foi caracterizada pela hipertrofia das células endoteliais nos sinusóides medulares e a histiocitose pela presença de macrófagos (histiócitos) (Figura 4).

A definição do padrão reatividade considerou a reação na zona cortical e paracortical do linfonodo, onde se situam respectivamente os linfócitos $\mathrm{B}$ e T. O padrão de reatividade foi considerado predominante se presente em 2/3 do número total de linfonodos reativos nestas áreas. A não preponderância de um tipo específico caracterizou o padrão de hiperplasia mista. O padrão não reativo foi definido como a falta de reatividade, inclusive hiperplasia sinusal, em mais de 2/3 do total de linfonodos.

Foi avaliada a presença de ruptura capsular do linfonodo, necrose tumoral e desmoplasia.

Todas as variáveis foram relacionadas com a recidiva da doença e entre si. Foi considerada igualmente a recidiva cervical, a distância ou o surgimento de um tumor primário.

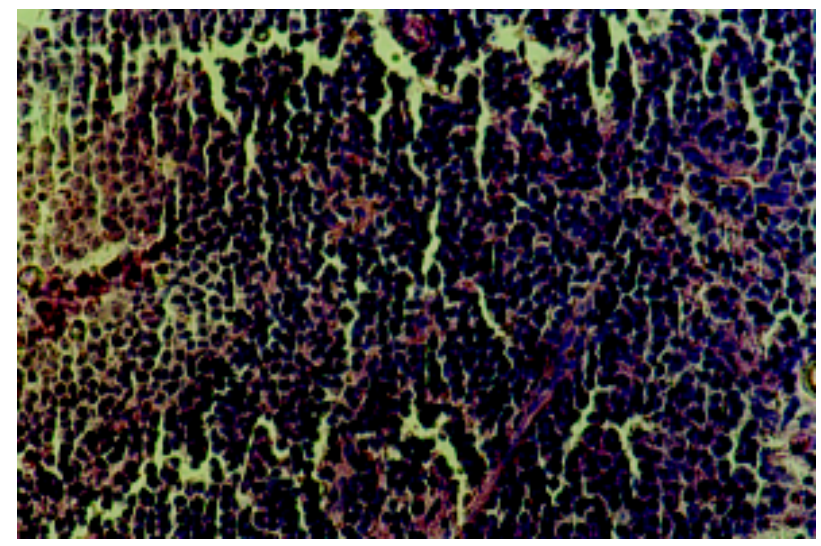

Figura 2 - Hiperplasia paracortical (HE, 125x).

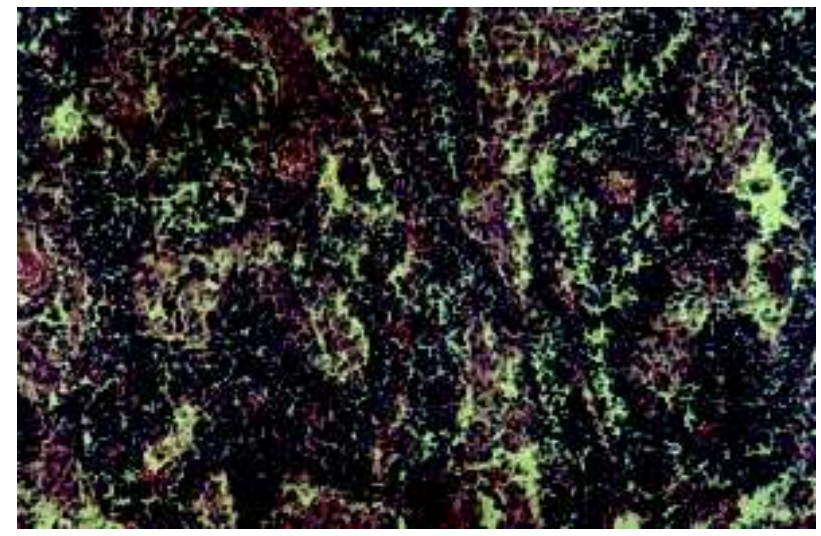

Figura 4 - Hiperplasia sinusal (HE, 125x), com histiocitose no detalhe (HE, 400x). 
A análise estatística empregou o teste exato de Fisher, considerando as diferenças com erro a inferior a $5 \%$.

\section{RESULTADOS}

Dezenove pacientes eram do sexo masculino e cinco do sexo feminino. A idade média foi de 55 anos (34 a 78). Treze pacientes desenvolveram recidiva da doença, 11 estavam assintomáticos por ocasião do último retorno ambulatorial. O seguimento médio dos pacientes assintomáticos foi de 52 meses (14 a 116). Oito pacientes apresentavam estadiamento $\mathrm{N}_{1}$ ou $\mathrm{N}_{2}$ e 15 pacientes estádio $\mathrm{N}_{3}$. Um paciente não pode ser reestadiado devido à falta da informação no prontuário.

Dezenove pacientes apresentavam carcinoma epidermóide e cinco carcinoma indiferenciado.

Foram estudados 601 linfonodos, média de 25 linfonodos por paciente (cinco a 50).

Do total de 601 linfonodos analisados, 463 (77\%) se mostraram reativos. Entre os reativos, 171 apresentavam hiperplasia paracortical, 174 hiperplasia folicular e os 118 restantes apresentavam hiperplasia sinusal.

Dezoito (75\%) pacientes apresentaram reatividade em mais de $2 / 3$ dos linfonodos.

Dez (55\%) entre 18 pacientes com predomínio de hiperplasia paracortical ou mista estavam assintomáticos, enquanto que apenas um $(16 \%)$ dos seis pacientes com predomínio de hiperplasia folicular ou padrão não reativo estavam livres de doença por ocasião do último retorno ambulatorial $(\mathrm{p}=0,11)$ (Tabela 1$)$.

Tabela 1

Reatividade linfonodal e recidiva da doença

\begin{tabular}{l|c|c}
\hline \multirow{2}{*}{} & \multicolumn{2}{|c}{ Recidiva } \\
\cline { 2 - 3 } & Sim & Não \\
\hline Hiperplasia paracortical & 4 & 5 \\
Hiperplasia mista & 4 & 5 \\
Hiperplasia folicular & 4 & 1 \\
Não reativo & 1 & 0 \\
\hline Total & 13 & 11 \\
\hline
\end{tabular}

Entre os oito pacientes com estádio $\mathrm{pN}_{1-2}$, todos apresentavam hiperplasia paracortical ou mista, e três (37\%) apresentaram recidiva. Entre os 15 pacientes $\mathrm{pN}_{3}$, nove apresentaram hiperplasia paracortical ou mista e quatro destes $(44 \%)$ desenvolveram recidiva.

Foi observada uma relação direta entre a presença de necrose tumoral e desmoplasia $(\mathrm{p}=0,002)$ (Tabela 2). Não foi evidenciada associação entre as outras variáveis.

Não foi observada relação entre as variáveis histiocitose, desmoplasia e necrose tumoral com a recidiva da doença (Tabela 3).

Os pacientes assintomáticos apresentaram em média 21 (cinco a 50) linfonodos no esvaziamento, enquanto en-
Tabela 2

Relação entre necrose tumoral e desmoplasia

\begin{tabular}{c|c|c}
\hline \multirow{2}{*}{} & \multicolumn{2}{|c}{ Desmoplasia } \\
\cline { 2 - 3 } & Sim & Não \\
\hline Necrose & & \\
Sim & 7 & 0 \\
Não & 5 & 12 \\
\hline
\end{tabular}

$p=0,002$

Tabela 3

Relação entre histiocitose, necrose e desmoplasia com a recidiva da doença

\begin{tabular}{l|r|r|r|c}
\hline & \multicolumn{2}{|c|}{ Recidiva } & & \\
\cline { 2 - 3 } & Sim & Não & Total & $p$ \\
\hline Histiocitose & 12 & 8 & 20 & 0,30 \\
$\quad$ Sim & 1 & 3 & 4 & \\
$\quad$ Não & & & & \\
Necrose & 3 & 4 & 7 & 0,65 \\
$\quad$ Sim & 10 & 7 & 17 & \\
$\quad$ Não & & & & \\
$\quad$ Desmoplasia & 6 & 6 & 12 & 1,0 \\
$\quad$ Sim & 7 & 5 & 12 & \\
$\quad$ Não & 13 & 11 & 24 & \\
\hline Total & 13 & & & \\
\hline
\end{tabular}

tre os pacientes com recidiva a média foi de 28 linfonodos (10 a 49). A presença de múltiplos linfonodos coalescentes, com seu parênquima quase totalmente substituído pelo tumor, impossibilitou a contagem precisa do número de linfonodos comprometidos.

\section{DISCUSSÃo}

A abordagem da reatividade linfonodal em pacientes com câncer apresenta problemas conceituais e técnicos. Entre estes citamos a distância entre o tumor e os diferentes linfonodos, a presença de infecções, o status nutricional do paciente e a heterogeneidade da resposta linfonodal. No presente estudo, o padrão de reatividade predominante foi determinado considerando córtex e paracórtex do linfonodo, área onde se situam os linfócitos. A hiperplasia sinusal, freqüentemente acompanhada de histiocitose, é considerada um padrão de reatividade não específico e tem sido encontrada também em situações de disfunção imunológica? .

A relação inversa entre histiocitose e a presença de metástase foi observada em outros estudos ${ }^{8,9}$. Esta relação poderia tanto representar uma resposta ativa dos histiócitos como ser apenas conseqüência do desenvolvimento tu- 
moral. As evidências apontam para a última hipótese, sendo relatado o desaparecimento progressivo do linfonodo à medida que aumenta o tamanho da metástase ${ }^{9}$.

Neste grupo, a hiperplasia paracortical se relacionou com maior índice de controle da doença, embora sem significância estatística, sugerindo que a resposta humoral seja pouco efetiva nestes carcinomas. Porém, não é possível afirmar se a resposta imune celular é efetiva ou se está associada com tumores de fenótipo menos agressivo.

No tumor primário oculto, a não existência de uma lesão ulcerada e infectada permite considerar a maior reatividade como uma resposta ao tumor.

O grande número de linfonodos reativos encontrado nos pacientes com tumor primário oculto contrasta com a agressividade da doença, freqüentemente em estádio $\mathrm{N}_{3}$ com ruptura capsular e substituição quase completa do linfonodo por tecido tumoral. A maior resposta imunológica observada nestes casos é uma evidência a favor da hipótese de regressão tumoral no sítio primário. Em melanomas foi observada regressão parcial do tumor primário por ocasião da metastatização linfática ${ }^{10}$, sugerindo que a metastatização possa ocasionar uma apresentação antigênica mais efetiva. Portanto, se faz necessária a caracterização das subpopulações de linfócitos T encontrados nas hiperplasias paracorticais, uma vez que a função de linfócitos CD4 ("T helper," e "T helper,") difere substancialmente entre si, como também dos linfócitos CD8 ( $\mathrm{T}$ citotóxico) e células natural killer (NK). Adicionalmente, deve ser considerada a presença de outras células, como macrófagos e células dendríticas, relacionadas com a ativação de linfócitos, apresentação antigênica e produção de citoquinas fibrogênicas, angiogênicas e imunossupressoras ${ }^{11,12}$.

A presença de necrose e desmoplasia, ambas indicadores de pior prognóstico, representa um parodoxo que começa a ser entendido ${ }^{13,14}$. A morte celular tumoral geralmente se dá por apoptose, uma forma de morte celular sem a liberação de mediadores inflamatórios ${ }^{15}$. A necrose poderia ocorrer por resposta imune ou por crescimento acelerado do tumor suplantando a neoangiogênese. Também sugere uma alteração dos mecanismos apoptóticos (p53, bax, bcl-2 ou outros) que deveriam ser acionados em condições isquêmicas. A necrose induz resposta inflamatória e poderia desencadear a fibroplasia local (desmoplasia). No processo de cicatrização, a deposição de colágeno se associa com a presença de citoquinas imunossupressoras e angiogênicas (IL-10 e TGF- $\beta)^{16,17}$. Esta modulação de citoquinas pode estar relacionada ao estímulo angiogênico necessário ao crescimento tumoral, bem como à produção de metaloproteinases relacionadas com a capacidade de invasão do tumor ${ }^{18}$. Recentemente foi demonstrado que tumores necróticos apresentam maior angiogênese, localizada em áreas adjacentes ao foco de necrose, também relacionada à presença de macrófagos ${ }^{19}$.

O tamanho da amostra foi pequeno para que a diferença observada nas taxas de recidiva alcançasse significância estatística (erro $\beta$ ); e também para considerar outros fatores prognósticos.

O uso da reatividade linfonodal como fator prognóstico esbarra na dificuldade de categorizar adequadamente diferentes pacientes com reatividade heterogênea. Outro fator a ser considerado é a remoção destes linfonodos. Não é possível afirmar que diante da persistência de doença residual, ou recidiva, seja desencadeada uma resposta semelhante no tecido linfóide remanescente.

A padronização e a reprodução destes resultados em amostras maiores são necessárias para que a reatividade linfonodal seja utilizada como indicador prognóstico. No momento, o maior interesse pelo assunto se concentra na compreensão da fisiologia tumoral. Concluímos que a reatividade linfonodal é freqüente e ocorre na maioria dos linfonodos regionais em pacientes com metástase cervical de tumor primário oculto e que a necrose tumoral está diretamente relacionada à presença de desmoplasia.

\begin{abstract}
Background: Our objetive is to assess the lymph node reactivity in patients with neck metastasis from occult primary tumor. Methods: We perform a retrospective analysis of 24 patients submitted to neck dissection between 1983 and 1995 due to metastasis of occult primary tumor. Slices of 601 resulting lymph nodes was colored with hematoxilin-eosin and evaluated by optical microscopy. Lymph node reactivity patterns considered the presence of paracortical hyperplasia, germinal center hyperplasia and sinus hyperplasia. We evaluated relationship between lymph node reactivity, capsular rupture, necrosis and desmoplasia with relapse of disease. Statiscal analyses were performed through a Fisher Test with 2 error less than 5\%. Results: Seventy seven percent of the lymph nodes were reactive. Fifty five percent of patients with paracortical or mixed hyperplasia and $16 \%$ of the patients with germinal center hiperplasia or non reactive lymph nodes were free of disease at the longest follow up $(p=0,11)$. The presence of necrosis and desmoplasia showed a significant relation $(p=0,02)$. Conclusions: The lymph node reactivity is usual in the great majority of primary unknown metastasis; tumoral necrosis is directly related with desmoplasia.
\end{abstract}

Key Words: Occult primary; Lymph nodes; Metastasis; Cancer. 


\section{REFERÊNCIAS}

1. Black MM, Kerpe S, Speer FD - Lymph node structure in patients with cancer of the breast. Am J Path, 1953; 24:505-21.

2. Bennet SH, Futrell JW, Roth JÁ - Prognostic significance of histologic host response in cancer of the larynx or hypopharynx. Cancer, 1971; 28:1255-65.

3. Tsakraklides V, Olson P, Kersey JH - Prognostic significance of the regional lymph node histology in cancer of the breast. Cancer, 1974; 34:1259-67.

4. Berlinger NT, Tsakraklides V, Pollak, K - Immunologic assessment of regional lymph node histology in relation to survival in head and neck carcinoma. Cancer, 1976; 37:697-705.

5. Bouquot JE, Weiland LH, Kurtland LT - Metastases to and from the upper aerodigestive tract in the population of Rochester, Minnesota, 1935-1984. Head Neck, 1989; 11:212-218.

6. Davidson BJ, Spiro RH, Patel S - Cervical metastases of occult origin: the impact of combined modality therapy. Am J Surg, 1994; 168: 395-9.

7. Rosai J \& Dorfman RF - Sinus histiocytosis with lymphadenopathy: a pseudolymphomatous benign disorder - Analysis of 34 cases. Cancer, 1972; 30:1174-88.

8. Amar A, Rapoport A, Rosas MP. Evaluation of lymph node reactivity in differentiated thyroid carcinoma. Sao Paulo Med J, 1999; 117: 125-8.

9. Berg, JW - Sinus histiocytosis: A fallacious measure of host resistance to cancer. Cancer, 1956; 9:935-9.

10. Ronan, SG; Eng, AM; Briele, HÁ - Thin malignant melanoma with regression and metastases. Arch Dermatol, 1987; 123:1326-30.

11. Herrmann G, Schumm-Draeger PM, Atai E. T lymphocytes, CD68-positive cells and vascularization in thyroid carcinomas. J Cancer Res Clin Oncol, 1994; 120: 651-6.

12. Young MRI, Wright M, Pandit R - Myeloid differentiation treatment to diminish the presence of immune-supressive CD34+ cells within human head and neck squamous cell carcinomas. J Immunol, 1997; 159: 990-6.

13. Noone, RB; Bonner Jr., H; Raymond, S - Lymph node metastases in oral carcinoma. Plast Reconstr Surg, 1974; 53:158-66.
14. Olsen, KD; Caruso, M; Foote, RL - Histopathologic predictors of recurrence after neck dissection in patients with lymph node invovement. Arch Otolaryngol Head Neck Surg, 1994; 120: 1370-74.

15. Mostafapour SP, Hockenbery DM, Rubel EW - Life and death in otolaryngology - Mecanisms of apoptosis and its role in the pathology and treatment of disease. Arch Otolaryngol Surg, 1999; 125:729-37.

16. Lee TY, Chin GS, Kim WJ - Expression of transforming growth factor beta 1, 2 and 3 proteins in keloids. Ann Plast Surg, 1999; 43: 179-84.

17. Conrad CT, Ernst NR, Dummer W - Differential expression of transforming growth factor beta 1 and interleukin 10 in progressing and regressing areas of primary melanoma. J Exp Clin Cancer Res, 1999; 18: 225-32.

18. Gohji K, Nomi M, Hara I - Influence of cytokines and growth factors on matrix metalloproteinase-2 production and invasion of human renal cancer. Urol Res, 1998; 26: 33-7.

19. Leek RD, Landers RJ, Harris AL - Necrosis correlates with high vascular density and focal macrophage infiltration in invasive carcinoma of the breast. Br J Cancer, 1999; 79:991-5.

Endereço para Correspondência:

Prof. Abrão Rapoport

Praça Amadeu Amaral, 47 - cj. 82

01327-010 - São Paulo - SP

E-Mail: cpcgcp.hosphel@attglobal.net 